\title{
Variability of peak expiratory flow rate in children: short and long term reproducibility
}

\author{
Thomas Frischer, Rolf Meinert, Radvan Urbanek, Joachim Kuehr
}

\begin{abstract}
Background - Variability of peak expiratory flow (PEF) has been proposed as a surrogate for bronchial hyperresponsiveness. The normal range of variability of PEF for children has been reported and the test has been used to screen for asthma in population based studies. However, there is little information on the reproducibility of the method in epidemiological settings.

Methods - In a cohort study of primary school children the variability in PEF was recorded in two consecutive years for one week (first survey) and two weeks (second survey) using mini Wright peak flow meters. PEF was recorded twice daily (morning and evening) and average amplitude as a percentage of mean was calculated as a standard measure of PEF variability for each single week of PEF measurement. Children with PEF variability exceeding the $90 \%$ percentile of the distribution for the specific time period were regarded as having increased variability of PEF.
\end{abstract}

Results - Of 66 children with increased PEF variability in the first year, 13 (19.7\%) had an abnormal test in the first week of the second year. Of 543 children with normal PEF variability in the first year, $44(8 \cdot 1 \%)$ had an abnormal test in the second study year (odds ratio $2 \cdot 8$, confidence interval (CI) $1 \cdot 4$ to $5 \cdot 4)$. Of 646 children in the second survey 61 (9.4\%) were abnormal during the first week and $68(10.5 \%)$ had an increased PEF variability during the second week, but only $24(3 \cdot 7 \%)$ children had an increased PEF variability in both weeks. The sensitivity (specificity) for doctordiagnosed asthma (12 month period prevalence) was $36.4 \%(91.0 \%)$ in the first week of the second survey. When measurements of both weeks of the second survey were used to calculate PEF variability there was little improvement in the sensitivity (38.1\%) and specificity $(91 \cdot 5 \%)$, mainly because of decreased compliance in the second measurement week.

Conclusions - In young children assessment of PEF variability in order to screen for asthma is of limited value because of the low reproducibility of the method.

(Thorax 1995;50:35-39)

Keywords: asthma, children, peak expiratory flow.
Asthma has been characterised as a disease where respiratory symptoms are based on a large variation of resistance to airflow over short periods of time. ${ }^{1}$ Airway inflammation has recently evolved as the central concept in the understanding of the disease. ${ }^{2}$ However, as long as reliable inflammatory markers which can be applied to field studies are not available, assessment of airway function in addition to anamnestic data from the patients' history remains the main instrument for characterising the disease.

Measurement of lung function by peak expiratory flow (PEF) using peak flow meters has provided epidemiologists in asthma research with an instrument which is particularly suitable for children. ${ }^{3-11}$ Many studies have been performed in which serial PEF measurements have been made to study the prevalence of asthma and asthma-like respiratory symptoms in both clinical ${ }^{6-810}$ and epidemiological ${ }^{3-5}$ settings. Protocols have varied considerably in different studies, measurements being made from twice a day ${ }^{45}$ up to every six hours, ${ }^{12}$ and children studied from one week ${ }^{12}$ up to one year. ${ }^{4}$ Parameters used have included determination of cosinor analysis, ${ }^{13}$ amplitude as percentage of maximum, ${ }^{3}$ coefficient of variation $^{4}$ or, most commonly, amplitude as percentage of mean..$^{3214}$ Conflicting evidence has been published regarding the sensitivity and specificity of PEF variability in detecting asthma. $^{3512}$ The rationale on which its value as an asthma screening test is based is the close association between PEF variability and nonspecific bronchial hyperreponsiveness as measured by histamine challenge. ${ }^{12}$

We have observed that variability in PEF has a low sensitivity for doctor-diagnosed asthma in young children..$^{15}$ We therefore hypothesised that a measurement period of one week, as used in our study, might have been too short and this may have been the main reason for our findings. The study was therefore repeated within the same cohort of children after one year and the observation period was extended to two weeks. The purpose of this report is to describe the long term variability (year to year) as well as the short term variability (week to week) of the measurement. Sensitivity and specificity of the one week protocol was compared with a two week protocol for a diagnosis of asthma.

\section{Methods}

DESIGN AND TIME SCHEDULE

This analysis is based upon data from a cohort study on the development of asthma and allergy 
in primary school children. Data collection took place between March 1990 and March 1993 at three locations in south-western Germany. The study aimed at investigating both genetic and environmental risk factors for the development of asthma, allergy, and bronchial hyperresponsiveness. Ethical approval was sought by the University of Freiburg and informed consent was obtained from the parents before all measurements. A detailed description of the study design is provided elsewhere. ${ }^{16}$ Briefly, parental questionnaires were distributed in the spring of each school year. The questionnaire was tested for validity and repeatability in a separate study. ${ }^{16}$ Home visits were performed between December and April in 1990-1 and 1991-2. Amongst other measurements (interview, dust sampling, urine collection) PEF recordings were performed using mini Wright peak flow meters in both years. In the spring of each study year skin prick tests were performed in order to define the atopic status of the children.

\section{QUESTIONNAIRE}

The 12 month period prevalence of asthma and recurrent wheezy bronchitis (defined as a doctor diagnosis) were recorded. A history of respiratory symptoms was also taken at each survey. For this report the 12 month period prevalences of respiratory symptoms recorded in the second (1991), third (1992), and fourth (1993) questionnaires were used for analysis in order to cover the time periods when PEF measurements had taken place. Cough after exercise was defined as an affirmative answer to "Did your child cough frequently after exercise or when exposed to cold air or fog in the last 12 months?" and wheeze as an affirmative answer to "In the last 12 months have you heard a whistling or wheezing noise in your child's breathing?" Morning cough was defined by the question "Did your child have a cough or shortness of breath at night or in the morning in the last 12 months?" A positive answer to "Did your child have attacks of shortness of breath or breathlessness in the last 12 months?" was taken to suggest dyspnoea.

\section{VARIABILITY IN PEF}

First survey (1990-1)

Children and their parents were instructed in the correct use of the mini Wright peak flow meter. Children were familiar with the use of the peak flow meter because an exercise test had taken place earlier in the study in which PEF recordings were performed. Parents were asked to record the best of three readings their child achieved twice daily (07.00-09.00 hours and 16.00-19.00 hours) and tests were performed while standing. Measurements were recorded on a specially designed sheet on which the verbal instructions were reinforced. The importance of adhering to the time at which measurements should be taken was emphasised and parents were encouraged to insert blanks rather than inaccurate data. Peak flow meters were collected after one week and sheets checked for plausibility of data. Current respiratory illness was defined as cough on more than two days during the study week.

\section{Second survey (1991-2)}

Home visits were repeated, including the same measurements as the year before. The same protocol and equipment were used for the collection of PEF data, but the observation period was extended to 14 days.

\section{SKIN PRICK TEST}

A standardised skin prick test using seven common allergens (birch, grass and hazel pollen, cat and dog dander, Dermatophagoides pteronyssinus and $D$ farinae (ALK Laboratories, Denmark)), a positive (histamine $10 \mathrm{mg} / \mathrm{ml}$ ) and a negative $(\mathrm{NaCl})$ control was performed on the right forearm of all children. The smallest and largest diameter of every weal was measured after 15 minutes and the arithmetic mean calculated. Sensitisation to a specific allergen was assumed when the allergen/histamine ratio was $\geqslant 0.5$ and the allergen weal was at least $2 \mathrm{~mm}$ in diameter. ${ }^{17}$ Children with at least one positive reaction to any of the allergens were designated as being atopic. The skin prick test was repeated at the second and third survey. For this analysis a child's atopic status was defined on the basis of the first and second skin prick test. A child was considered to be atopic when either test was positive.

\section{DATA ANALYSIS}

As an index of PEF variability the ratio of amplitude/mean of daily PEF expressed as a percentage (amplitude/mean as a percentage $=$ (higher value - lower value)/mean $\times 100$ ) was calculated for each day. Diurnal variability of PEF was calculated as the average amplitude/ mean as a percentage for the observation time for those children who had measured their PEF twice daily for at least five days at the first survey. At the second survey PEF variability was calculated in the same manner but for the two measurement weeks separately. A further measure of PEF variability was derived using the PEF data from both weeks at the second survey for children with at least 10 days of complete data. For each of the time periods investigated children whose $\mathrm{PEF}$ variability exceeded the upper $90 \%$ percentile of the specific distribution of average amplitude as a percentage of mean were classified as having an increased variability of PEF. Because of the skewed distribution of average amplitude as a percentage of mean, correlations between time periods were assessed using Spearman's rank correlation coefficient. Associations with health variables were cross tabulated. All analyses were performed using the Statistical Analysis System (SAS).

\section{Results}

Of 1303 families visited during the first survey, 1237 (94.9\%) agreed to undergo PEF measurements. The mean (SD) age of the children 
at study entry was $7 \cdot 3$ years $(0 \cdot 4)$ years. Of these families, complete data sets - that is, $\mathrm{PEF}$ measurements twice daily for five days - could be obtained for $991(80 \cdot 1 \%)$ children. At the second survey 1206 households were visited and $1026(85 \cdot 1 \%)$ performed PEF measurements. A total of $847(82.6 \%)$ children during the first week and $725(70.7 \%)$ during the second week performed PEF manoeuvres twice daily for five days; $764(74.5 \%)$ children had 10 days of complete measurements (table 1). The 12 month period prevalence of asthma was $1.3 \%$ at the first survey and $1 \cdot 2 \%$ at the second survey. A diagnosis of recurrent wheezy bronchitis was recorded in $2.0 \%$ of children in the first survey and $2 \cdot 5 \%$ in the second survey.

\section{SHORT TERM REPRODUCIBILITY}

The Spearman correlation coefficient for average amplitude as a percentage of mean between the first and the second week of the second survey was 0.49 ( $p<0.001$; fig 1 ) for all participants, $0.34(p<0.05)$ for children with asthma at either survey, and $0.5(p<0.001)$ for children with respiratory symptoms suggestive of asthma (at least one of four respiratory symptoms) at either survey. The association improved marginally when children with respiratory symptoms during the study period were excluded $(r=0.53 ; \mathrm{p}<0.001)$. In order to investigate whether this finding is due to a low correlation in children with low PEF variability, values of average amplitude as a percentage of mean of the first study week were divided into quartiles and quartile specific correlations were calculated, but these did not explain the low correlation (1st quartile: $r=0 \cdot 28$, 2nd quartile: $r=0 \cdot 13$, 3rd quartile: $r=0 \cdot 15$; 4th quartile: $r=0 \cdot 4)$. Of 646 children who performed five days of PEF measurements both in the first and the second week, $61(9.4 \%)$ were abnormal in the first week, 68 $(10.5 \%)$ in the second week, and $24(3.7 \%)$ in both weeks.

\section{LONG TERM REPRODUCIBILITY}

The correlation of average amplitude as a percentage of mean between the study years was low (fig 2) regardless of the time period used to calculate average amplitude as a percentage of mean $(r=0.2$ for first year $v$ first week of second year; $r=0.15$ for first year $v$ second week of second year; $r=0.2$ for first year $v$ first and second week of second year). When calculated for various subgroups (see above) the associations did not improve (data not shown), indicating that a major change in the

Table 1 Statistics of PEF data in the two surveys

\begin{tabular}{|c|c|c|c|c|}
\hline & \multirow[t]{2}{*}{ First survey } & \multicolumn{3}{|c|}{ Second survey } \\
\hline & & Week $1^{\mathrm{a}}$ & Week $2^{a}$ & Weeks $1+2^{\mathrm{b}}$ \\
\hline $\begin{array}{l}\mathrm{n} \\
\text { Mean average amplitude/mean } \\
(\%)\end{array}$ & $\begin{array}{l}991 \\
7 \cdot 3\end{array}$ & $\begin{array}{l}847 \\
7 \cdot 0\end{array}$ & $\begin{array}{c}725 \\
6.7\end{array}$ & $\begin{array}{l}764 \\
6 \cdot 8\end{array}$ \\
\hline $\begin{array}{l}\text { Median (\%) } \\
90 \% \text { percentile (\%) }\end{array}$ & $\begin{array}{r}6 \cdot 3 \\
12 \cdot 4\end{array}$ & $\begin{array}{r}5 \cdot 9 \\
12 \cdot 7\end{array}$ & $\begin{array}{r}5 \cdot 5 \\
12 \cdot 2\end{array}$ & $\begin{array}{r}5 \cdot 9 \\
11 \cdot 6\end{array}$ \\
\hline
\end{tabular}

At least 5 of 7 days with complete data.

b At least 10 of 14 days with complete data.

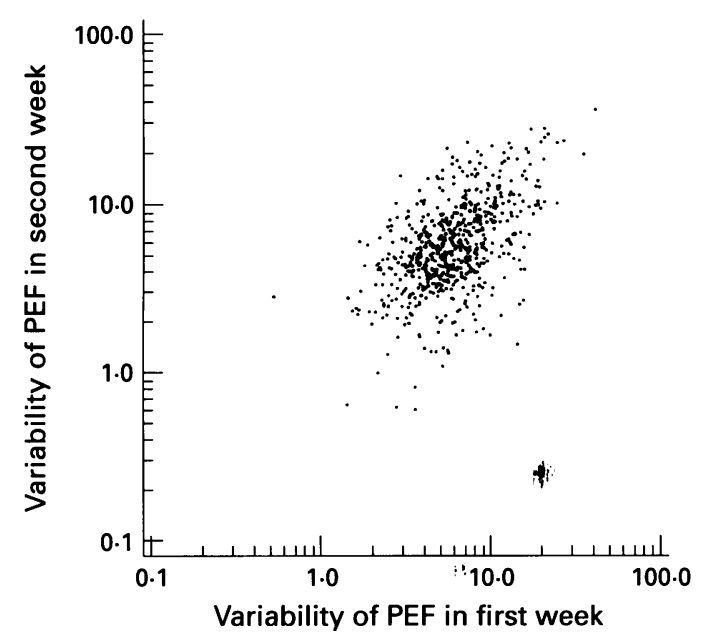

Figure 1 Week to week variability of diurnal variability of peak expiratory flow. Variability of $P E F=$ average amplitude/mean as a percentage for all children who had measured their PEF twice daily for at least five days in both measurement weeks of the second survey $(n=646)$. Scale is logarithmic.

respiratory illness of the children had not accounted for our findings. Of 66 children with increased average amplitude as a percentage of mean in the first year, $13(19 \cdot 7 \%)$ had an abnormal test in the first week of the second year. Of 543 children with normal PEF variability in the first year, $44(8 \cdot 1 \%)$ had abnormal tests in the second study year. Children with an increased PEF variability therefore had a 2.8 fold risk (confidence interval (CI) 1.4 to $5 \cdot 4, p=0.002)$ for an increased PEF variability after one year compared with children with a normal variability in PEF.

\section{ASSOCIATION WITH HEALTH VARIABLES}

The associations between health variables and PEF variability were similar in the first survey and the first week of the second survey, but somewhat weaker in the second week of the second survey (table 2). Sensitivity (specificity) of the test for a diagnosis of asthma (12 month

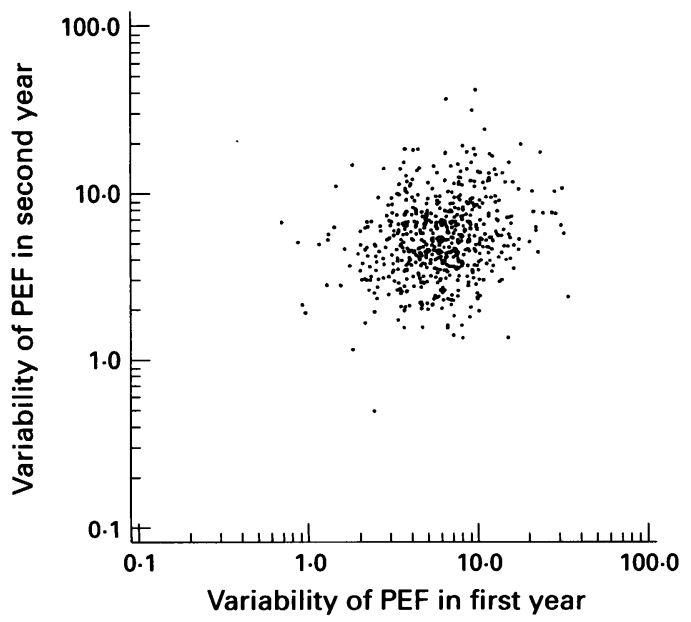

Figure 2 Year to year variability of diurnal variability of peak expiratory flow. Variability of $P E F=$ average amplitude/mean as a percentage for all children who had measured their PEF twice daily for at least five days of the first survey and also for at least five days during the first week of the second survey $(n=609)$. Scale is logarithmic. 
Table 2 Assocation of diurnal variability of PEF $>90 \%$ percentile with respiratory health variables. Values are percentages

\begin{tabular}{|c|c|c|c|c|}
\hline & \multirow{2}{*}{$\begin{array}{l}\text { First survey } \\
\text { (1991) }\end{array}$} & \multicolumn{3}{|c|}{ Second survey (1992) } \\
\hline & & Week 1 & Week 2 & Weeks $1+2^{a}$ \\
\hline $\begin{array}{l}\text { Asthma }{ }^{b} \\
\text { No asthma } \\
\text { Recurrent wheezy bronchitis } \\
\text { Asthma }\end{array}$ & $\begin{array}{r}8 \cdot 8 \\
41 \cdot 7 \\
38 \cdot 9\end{array}$ & $\begin{array}{r}8 \cdot 7 \\
40 \cdot 0 \\
36 \cdot 4\end{array}$ & $\begin{array}{r}8 \cdot 4 \\
50 \cdot 0 \\
26 \cdot 3\end{array}$ & $\begin{array}{c}8 \cdot 1 \\
44 \cdot 4 \\
38 \cdot 1\end{array}$ \\
\hline $\begin{array}{l}\text { No. of respiratory symptoms } \\
0 \\
1 \\
2 \\
3 \\
4\end{array}$ & $\begin{array}{r}7 \cdot 7 \\
9 \cdot 5 \\
19 \cdot 3 \\
25 \cdot 0 \\
50 \cdot 0\end{array}$ & $\begin{array}{r}8 \cdot 8 \\
9 \cdot 5 \\
12 \cdot 8 \\
22 \cdot 2 \\
28 \cdot 6\end{array}$ & $\begin{array}{r}8 \cdot 2 \\
13 \cdot 3 \\
11 \cdot 1 \\
10 \cdot 5 \\
16 \cdot 7\end{array}$ & $\begin{array}{r}7 \cdot 7 \\
11 \cdot 9 \\
16 \cdot 7 \\
13 \cdot 6 \\
33 \cdot 3\end{array}$ \\
\hline $\begin{array}{l}\text { Skin prick test positive } 1990 \text { or } \\
1991 \\
\text { No } \\
\text { Yes }\end{array}$ & $\begin{array}{r}9 \cdot 3 \\
11 \cdot 8\end{array}$ & $\begin{array}{r}8 \cdot 7 \\
13 \cdot 2\end{array}$ & $\begin{array}{r}9 \cdot 6 \\
12 \cdot 4\end{array}$ & $\begin{array}{r}9 \cdot 1 \\
12 \cdot 3\end{array}$ \\
\hline $\begin{array}{l}\text { Current respiratory illness } \\
\text { during study period } \\
\text { No } \\
\text { Yes }\end{array}$ & $\begin{array}{r}9 \cdot 7 \\
11 \cdot 8\end{array}$ & $\begin{array}{r}9 \cdot 6 \\
14 \cdot 3\end{array}$ & $\begin{array}{r}9 \cdot 9 \\
10 \cdot 1\end{array}$ & $\begin{array}{r}9 \cdot 7 \\
12 \cdot 2\end{array}$ \\
\hline
\end{tabular}

10 of 14 days with complete PEF data.

${ }^{b} 12$ month period prevalence. to study the prevalence of asthma in children. It requires simple equipment, is well tolerated by children, and it has been shown that PEF variability is closely associated with non-specific bronchial hyperresponsiveness as assessed by histamine challenge. ${ }^{12}$ We observed a similar relationship between doctor diagnosed asthma and PEF variability on the one hand, and recurrent wheezy bronchitis and PEF variability on the other, supporting the view that recurrent wheezy bronchitis and asthma are both part of a single disease spectrum. Quackenboss et al studied a sample of adults and 208 children (6-15 years) in whom PEF recordings were performed four times a day over two weeks. ${ }^{3}$ They reported a sensitivity of amplitude as percentage of mean to detect asthma of $60 \%$ but a specificity of $76.8 \%$. In our study the sensitivity was $39 \%$ in the first survey.

The children used in our study were derived from a longitudinal study. They had participated in an exercise test prior to the PEF study and were used to the peak flow meter. ${ }^{15}$ Moreover, household visits were performed more often in better educated families who were more interested in the study. We therefore assume that our differing results are not based merely on poor compliance. Reasons for the differences in our findings compared with those of Quackenboss et al may relate to their use of four times daily measurements, a cutoff point that yielded a high sensitivity but low specificity, and a different population studied (asthma prevalence of about $20 \%$ compared with $2.5 \%$ in our study). Our study shows that there are limitations to the method which can be classified into several components: (1) decreasing compliance with longer measurement periods thus introducing measurement error (particularly in an unselected sample of healthy children); (2) biological variability of bronchial hyperresponsiveness; and (3) biological meaning of increased PEF variability which may differ from other surrogates used to estimate the degree of bronchial hyperresponsiveness.

Measurement error can be introduced by a decreasing compliance over the study period. In our study more missing values occurred in the second week and there was a tendency for the test to be performed less well in the second week. Consequently, an extension of the measurement period did not increase the qualities of the test if the aim was to use assessment of PEF variability as an asthma screening test in a healthy paediatric population. Additionally, PEF in children has been shown to be a parameter which is less reproducible than, for example, $\mathrm{FEV}_{1}$. Strachan studied the repeatability of lung function parameters in seven year old children ${ }^{18}$ and observed that, for two repeated PEF measures within subjects, as much as $34 \%$ of the variance was attributable to measurement error.

Bronchial hyperresponsiveness as measured by direct or indirect stimuli varies considerably over time, ${ }^{19}$ reflecting the natural course of asthma, environmental influences such as passive smoking, ${ }^{20}$ or an inherent variability of bronchial hyperresponsiveness. In non-selected population based studies of children only a

\section{Discussion}

Assessment of variability of PEF by home measurements using the mini Wright peak flow meter has been proposed as an attractive tool 
loose relationship has been observed between PEF variability and the prevalence or severity of asthma. ${ }^{421}$ Characterisation of a subject on the basis of a single test for bronchial hyperresponsiveness has been questioned, ${ }^{21}$ and emphasis placed on repeated measurements. ${ }^{19} \mathrm{Our}$ findings suggest that within a two week period only about one of three children with increased PEF variability in one week have a similar degree of PEF variability in the second week. Hence, assessment of PEF variability by twice daily measurement of PEF is influenced by considerable random variability which impairs its qualities as an asthma screening test in small children.

Apart from the absolute level of agreement of measurement over a short period of time, a second aspect of reproducibility applies to the relative stability of ranking of individuals in a population over longer observation periods. The absolute values might change in a population over time - for example, as a result of age and other factors - influencing PEF variability. The correlation between different measurements is a measure of relative rather than of absolute reproducibility. In our study the reproducibility over a one year period was low even when looked upon as "relative" reproducibility.

Cockroft argued that variability of PEF reflects underlying non-specific bronchial hyperresponsiveness which has to be assessed in the temporal relationship to respiratory symptoms. ${ }^{2}$ We have no detailed data on current respiratory symptoms suggestive of asthma, but investigated the relationship between the 12 month period prevalence of these symptoms and PEF variability. We observed a "doseresponse-like" association between the number of respiratory symptoms and the average amplitude as a percentage of mean, $30-50 \%$ of children with four symptoms showing an increased variability in PEF. Of children without symptoms suggestive of asthma, only $7 \cdot 7-8 \cdot 8 \%$ had an increased average amplitude as a percentage of mean. Thus, assessment of PEF variability might help in determining whether respiratory symptoms in children, as reported by parents, are based on an underlying bronchial hyperresponsiveness. Furthermore, increased variability of PEF might constitute a risk factor for respiratory symptoms later on. In our study children with increased PEF variability at either survey had an increased risk for developing symptoms subsequently, irrespective of their current symptom status.

Bronchial hyperresponsiveness, as assessed by direct or indirect stimuli, has been reported to be closely connected to atopy, whether defined on the basis of IgE status ${ }^{22}$ or the results of skin prick tests. ${ }^{23}$ However, in our study there was a very weak association between variability of PEF and atopy. One explanation might relate to the presence of allergic inflammation in the airways which can be better measured by indirect challenge tests than by PEF variability.

1 Scadding JG. Definition and clinical categorisation. In: Weiss EB, Segal MS, eds. Bronchial asthma: mechanisms and therapeutics. Boston: Little, Brown, 1976:19-30.

2 Cockcroft DW. Airway hyperresponsiveness. Therapeutic implications. Ann Allergy 1987;59:405-14.

3 Quackenboss JJ, Lebowitz MD, Krzyzanowski M. The normal range of diurnal changes in peak expiratory flow rates. Relationship to symptoms and respiratory disease. $\mathrm{Am}$ Relationship to symptoms and
Rev Respir Dis 1991;143:323-30.

4 Josephs LK, Gregg I, Mullee MA, Holgate ST. Nonspecific bronchial reactivity and its relationship to the clinical expression of asthma. A longitudinal study. Am Rev Respir Dis 1989;140:350-7.

5 Siersted HC, Hansen HS, Hansen NCG, Hyledbrandt N, Mostgaard G, Oxhoj H. Evaluation of peak expiratory flow variability in an adolescent population sample: the Odense schoolchild study. Am $\mathcal{f}$ Respir Crit Care Med

6 Bellia V, Visconti A, Insalaco G, Cuttitta G, Ferrara G, Bonsignore $G$. Validation of morning dip of peak expiratory flow as an indicator of the severity of nocturnal asthma. Chest 1988;94:108-10.

7 Sly PD, Hibbert ME, Landau LI. Diurnal variation of peak expiratory flow rate in asthmatic children. Pediatr Pulmonol 1986;2:141-6.

8 Albertini M, Politano S, Berard E, Boutte P, Mariani R. Variation in peak expiratory flow of normal and asymptomatic asthmatic children. Pediatr Pulmonol 1989;7:140-4

9 Sanz J, Martorell A, Saiz R, Alvarez V, Carrasco JI. Peak expiratory flow measured with the mini Wright peak flow meter in children. Pediatr Pulmonol 1990;9:86-90.

10 Henderson AJ, Carswell F. Circadian rhythm of peak expiratory flow in asthmatic and normal children. Thorax 1989;44:410-4.

11 Johnston I, Anderson HR, Patel S. Variability of peak flow in wheezy children. Thorax 1984;39:583-7.

12 Ryan G, Latimer KM, Dolovich J, Hargreave FE. Bronchial responsiveness to histamine: relationship to diurnal variation of peak flow rate, improvement after bronchodilator, and airway caliber. Thorax 1982;37:423-9.

13 Hetzel MR, Clarke TJH. Comparison of normal and asthmatic circadian rhythms in peak expiratory flow rates. Thorax 1980;35:732-8.

14 Higgins BG, Britton JR, Chinn S, Jones TD, Jenkinson D, Burney PGJ, et al. The distribution of peak expiratory flow variability in a population sample. Am Rev Respir Dis 1989;140:1368-72.

15 Frischer T, Kuehr J, Meinert R, Karmaus W, Forster H, Urbanek R. Relationship between response to exercise and diurnal variability of peak expiratory flow rate in primary school children. Thorax 1993;48:249-53.

16 Frischer T, Kuehr J, Meinert R, Karmaus W, Urbanek R. Risk factors for childhood asthma and recurrent wheezy bronchitis. Eur f Pediatr 1993;152:771-5.

17 Meinert R, Frischer T, Karmaus W, Kuehr J. Influence of skin prick test criteria on estimation of prevalence and incidence of allergic sensitization in children. Allergy 1994; 49:526-32.

18 Strachan DP. Repeatability of ventilatory function measurements in a population survey of 7 year old children. Thorax 1989;44:474-9.

19 Redline S, Tager IB, Speizer FE, Rosner B, Weiss ST. Longitudinal variability in airway responsiveness in a population-based sample of children and young adults. Intrinsic and contributing factors. Am Rev Respir Dis 1991; 144:507-12.

20 Frischer T, Kuehr J, Meinert R, Karmaus W, Urbanek $R$. Influence of maternal smoking on variability of peak expiratory flow rate in school children. Chest 1993; 104: 1133-7.

21 Pattemore PK, Asher MI, Harrison AC, Mitchell EA, Rea $\mathrm{HH}$, Stewart AW. The interrelationship among bronchial hyperresponsiveness, the diagnosis of asthma and asthma hyperresponsiveness, the diagnosis of asthma and
symptoms. Am Rev Respir Dis 1990;142:549-54.

22 Sears MR, Burrows B, Flannery EM, Herbison GP, Hewitt CJ, Holdaway MD. Relation between airway responsiveness and serum IgE in children with asthma and in apparently normal children. $N$ Engl f Med 1991;325: 1067-71.

23 Studnicka M, Frischer T, Weiss ST, Dockery D, Speizer F, Neumann $M$. Seasonal and allergenic predictors of bronchial responsiveness to distilled water. Am Rev Respir Dis 1993;148:1460-6. 